\title{
Classification of Surface Electromyographic Signals using AM-FM Features
}

\author{
Christodoulos I. Christodoulou, Prodromos A. Kaplanis, Victor Murray, Member IEEE, Marios S. \\ Pattichis, Senior Member IEEE and Constantinos S. Pattichis, Senior Member IEEE
}

\begin{abstract}
The objective of this study was to evaluate the usefulness of AM-FM features extracted from surface electromyographic (SEMG) signals for the assessment of neuromuscular disorders at different force levels. SEMG signals were recorded from a total of 40 subjects, 20 normal and 20 patients, at $10 \%, 30 \%, 50 \%, 70 \%$ and $100 \%$ of maximum voluntary contraction (MVC), from the biceps brachii muscle. From the SEMG signals, we extracted the instantaneous amplitude, the instantaneous frequency and the instantaneous phase. For each AM-FM feature their histograms were computed for 32 bins. For the classification, three classifiers were used: (i) the statistical K-nearest neighbour (KNN), (ii) the neural self-organizing map (SOM) and (iii) the neural support vector machine (SVM). For all classifiers the leave-one-out methodology was implemented for the classification of the SEMG signals into normal or pathogenic. The test results reached a classification success rate of $80 \%$ when a combination of the three AM-FM features was used.
\end{abstract}

Index Terms-AM-FM, SEMG, classification

\section{INTRODUCTION}

$\mathrm{T}_{\mathrm{i}, \mathrm{s}}^{\mathrm{H}}$ electromyographic (EMG) examination provides important information for the assessment of neuromuscular disorders and is generally carried out using needle electrodes. However needle electrodes put the patients under considerable pain and discomfort, as well as the risk of infection. In addition, constant expert supervision is necessary, which renders the whole process quite lengthy. In pediatric examinations and tests in particular, there are even more difficulties in using needle electrodes, and long term monitoring is quite difficult, [1]. Surface electrodes and the acquisition of surface EMG signals provide a noninvasive alternative to needle EMG for the detection of neuromuscular disorders. At present a surface detected signal is preferred only for obtaining "global" information about the time and/or intensity of superficial muscle activation [1].

In previous work [2]-[6] it was shown that time and frequency domain features extracted from the EMG signals can be used successfully for the classification and the identification of neuromuscular disorders. Abel et al [3], found a percentage of correct classifications of $75 \%$ when 12

C.I. Christodoulou, P.A. Kaplanis and C.S. Pattichis are with the Department of Computer Science, University of Cyprus, P.O. Box 20537, Nicosia, Cyprus (e-mail: cschr2@ucy.ac.cy, p.a.kaplanis@cytanet.com.cy, pattichi@ucy.ac.cy)

V. Murray and M.S. Pattichis are with the Department of Electrical and Computer Engineering, University of New Mexico, Albuquerque, NM 87131, USA (e-mail: vmurray@ieee.org, pattichis@ece.unm.edu). normal subjects were compared with 18 myopathy and 15 neuropathy patients examined with needle EMG. Turns analysis and small segments analysis were used. However authors concluded that the classification methods used, did not offer better results than the Interference Pattern analysis and could not by any means match the diagnostic success of an experienced clinician. Christodoulou et al [4] developed a modular neural networks system where multiple features extracted from needle EMG signals were fed into multiple classifiers for the assessment of 12 normal subjects, 13 subjects suffering with myopathy and 13 subjects suffering with motor neuron disease, reaching a diagnostic yield of $87.5 \%$. Abou-Chadi et al [5] used three versions of ANN to facilitate automatic classification of SEMG. With unsupervised techniques, the correct classification score reached $80 \%$, when five normal subjects and five myopathy subjects were selected from a pool of 14 normal subjects and 14 patients. Recordings were performed for 5 seconds at $50 \%$ MVC. Abou-Chadi et al [5] reached the conclusion that when SEMG is properly processed, it may provide the physician with a diagnostic assisting tool. In another SEMG study Kaplanis [6] reached a correct classification score of $82.9 \%$, when 91 control subjects and 20 pathogenic cases were placed in a pool of subjects. One may comment that this result may be misleading due to a higher number of control subjects as compared with pathogenic cases, however normalisation based on the number of subjects for each group during the classification process was performed.

In this work, we investigate new feature sets extracted using the AM-FM representations. Three sets of AM-FM features were estimated from the SEMG signals at various force levels: (i) the instantaneous amplitude, (ii) the instantaneous frequency, and (iii) the instantaneous phase. For each AM-FM estimate, we computed the histograms and used them as inputs to the classifiers. For the classification, three classifiers were implemented: (i) the statistical Knearest neighbour $(\mathrm{KNN})$ classifier, (ii) the neural selforganizing map (SOM) and (iii) the neural support vector machine (SVM). For all classifiers the leave-one-out methodology was implemented for the classification of the SEMG signals into two classes, i.e. normal or pathogenic.

\section{MATERIAL AND DATA ACQUiSITION}

Surface EMG recordings were acquired from 20 control subjects (NOR) and 20 neuromuscular subjects, from which 11 suffered from myopathy and 9 from neuropathy. Patients referred were first examined and diagnosed by their physician and were divided according to the general type of neuromuscular disorder (myopathy or neuropathy). 
Recording took place at a special Electromyography / Electroencephalography / Evoked Potential (EMG/EEG/EP) lab, with controlled temperature and humidity at the Department of Clinical Neurophysiology at the Cyprus Institute of Neurology and Genetics, Nicosia, Cyprus [6]. The Nicolet Viking IV electromyography a two-channel amplifier used unit was according to manufacturers specifications, fully electrically isolated to IEC 601-1 and BSS 5724, Part 1 Type BF. The input impedance of the system, Zin was stated to be $>1000 \mathrm{M} \Omega$. Through the system the low and high frequency values for recording were set at 20 and $500 \mathrm{~Hz}$ respectively.

A calibrated force measurement system, with a total weight of $40 \mathrm{~kg}$ was placed at the foot end of a couch, used for the subjects to lie down. The weights were lifted via a strap placed at the subjects' wrist and connected to the system through a force transducer, which was connected directly to a calibration circuit. Then the subject was required to pull at maximum voluntary contraction (MVC) three times with an interval of two minutes in between. A note of the MVC was made on the oscilloscope with a red tape. Recordings were made at five different force levels, i.e. at $10 \%, 30 \%, 50 \%, 70 \%$ and $100 \%$ of MVC from the biceps brachii muscle generated under isometric voluntary contraction (IVC).

\section{FEATURE EXTRACTION}

\section{A. AM-FM models}

Amplitude-modulation frequency-modulation (AM-FM) models [7], [8] allow us to model non-stationary signal content in terms of a series expansion of AM-FM component signals. AM-FM models have been used in a variety of applications including image reconstruction [7], image retrieval and video segmentation. Recently, some of the underlying theory of multidimensional frequency modulation was reported in [8].

Using a multi-scale filter bank, for any given signal $f(k)$, we compute a one-dimensional, single scale analytic signal, as given by [7]:

$$
f_{A S}(k)=f(k)+j H\{f(k)\},
$$

where $H\{$.$\} denotes the one-dimensional Hilbert transform$ operator applied along the signal. We estimate the instantaneous amplitude (IA), the instantaneous phase (IP) and the instantaneous frequency (IF) of the signal using

$$
\begin{aligned}
& a(k)=\left|f_{A S}(k)\right|, \\
& \varphi(k)=\arctan \left(\frac{\left.\operatorname{imag}\left(f_{A S}(k)\right)\right)}{\operatorname{real}\left(f_{A S}(k)\right)}\right) \\
& \varphi_{k}(k)=\frac{\partial \varphi(k)}{\partial k} \cong \frac{1}{n} \arccos \left(\frac{f_{A S}(k+n)+f_{A S}(k-n)}{2 f_{A S}(k)}\right) .
\end{aligned}
$$

where in (4), $n$ is a variable displacement from 1 to 4 , based on the argument that provides the minimum condition number to arcos function.

From the two generated estimates, the histograms for 32 equal width bins were computed and were used as input feature sets for classification. The histograms were further normalized by division of the histogram with the number of SEMG signal points in order to alleviate any bias due to different signals lengths.

\section{ClasSifiCATION}

The three AM-FM histograms (i.e. 96 bins) were used as one feature vector and were inputted into the following three classifiers. Furthermore the leave-one-out methodology was applied where for each input pattern to be classified, all the remaining patterns were used as the training set. The average of all classifications scores was the final score. This made the classification procedure independent of bootstrap sets and the results more robust and reliable.

\section{A. The KNN Classifier}

The statistical k-nearest neighbor (KNN) classifier [9] was used for the classification of the SEMG signals using the leave-one-out methodology. In the KNN algorithm, in order to classify a new input pattern, its $k$ nearest neighbors from the training set were identified. The new pattern was classified to the most frequent class among its neighbors based on a similarity measure that is usually the Euclidean distance. In this work the KNN system was implemented for several values of $k(k=1,3,5,7,9,11,13$ and 15) and it was tested using for input the three AM-FM histograms at the different force levels.

\section{B. The SOM Classifier}

The SOM was chosen because it is an unsupervised learning algorithm where the input patterns are freely distributed over the output node matrix [10]. The weights are adapted without supervision in such a way, so that the density distribution of the input data is preserved and represented on the output nodes. This mapping of similar input patterns to output nodes, which are close to each other, represents a discretisation of the input space, allowing a visualization of the distribution of the input data. The output nodes are usually ordered in a two dimensional grid and at the end of the training phase, the output nodes are labeled with the class of the majority of the input patterns of the training set, assigned to each node.

In the evaluation phase, a new input pattern was assigned to the winning output node with the weight vector closest to the new input vector. In order to classify the new input pattern, the majority of the labels of the output nodes in an $R x R$ neighborhood window centered at the winning node, were considered. The number of the input patterns in the neighborhood window for the two classes $m=\{1,2\}$, (1=normal, $2=$ pathogenic), was computed as:

$$
S N_{m}=\sum_{i=1}^{L} W_{i} N_{m i}
$$

where $L$ is the number of the output nodes in the $R \times R$ neighborhood window with $L=R^{2}$ (e.g. $L=9$ using a $3 \times 3$ window), and $N_{m i}$ is the number of the training patterns of the class $m$ assigned to the output node $i$. 

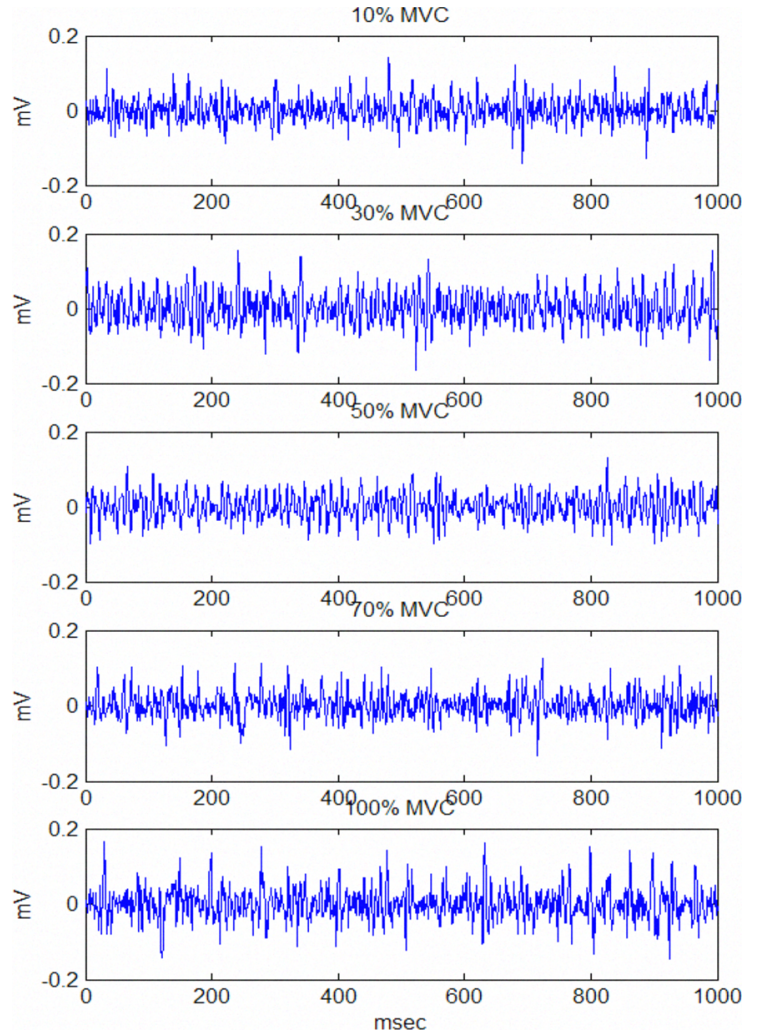
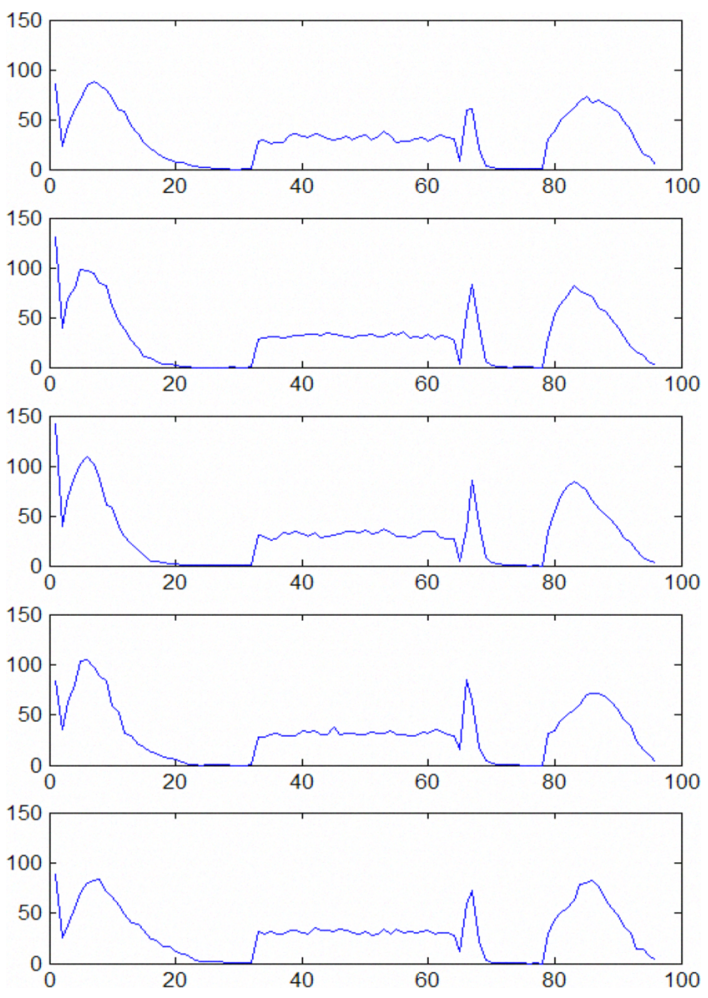

Fig. 1. Samples of SEMG signals from a normal subject and their corresponding AM-FM histograms per force level.

$W_{i}=1 /\left(2 d_{i}\right)$, is a weighting factor based on the distance $d_{i}$ of the output node $i$ to the winning output node. $W_{i}$ gives the output nodes near to the winning output node a greater weight than the ones farther away (e.g. in a $3 \times 3$ window, for the winning node $W=1$, for the four nodes perpendicular to the winning node $W_{i}=0.5$ and for the four nodes diagonally located $W_{i}=0.3536$, etc). The evaluation input pattern was classified to the class $m$ of the $S N_{m}$ with the greatest value, as normal or pathogenic.

\section{The SVM Classifier}

The Support Vector Machine (SVM) was also used for developing classification models for the problem. The method is initially based on a nonlinear mapping of the initial data set using a function $\varphi($.$) and then the$ identification of a hyperplane which is able to achieve the separation of two categories of data. Details about the implementation of the SVM algorithm used can be found in [11]. The SVM network was implemented using Gaussian Radial Basis Function (RBF) kernels; this was decided, as the rest of the kernel functions could not achieve satisfactory results.

\section{Combining}

From each subject five feature vectors were calculated one for each force level. Figure 1 shows samples of SEMG signals from a normal subject and their corresponding AMFM histograms per force level (only shown 1000 samples points for visibility).
The five classification outputs per subject were further combined using majority voting, i.e. the subject was assigned to the class where the majority of the five individual SEMG signals per force level were assigned. This was done in order to get a final and more reliable estimate of the classification result, since as it was shown in [4], modular neural networks system enhanced the diagnostic performance of the individual ANN classifiers making the whole system more robust and reliable.

\section{RESULTS}

Surface EMG recordings from 20 control subjects (NOR) and 20 neuromuscular subjects (11 myopathy (MYO) and 9 neuropathy (NEURO)) were recorded at $10 \%, 30 \%, 50 \%$, $70 \%$ and $100 \%$ of maximum voluntary contraction (MVC), from the biceps brachii muscle. For each SEMG recording the AM-FM features instantaneous amplitude (IA), instantaneous phase (IP), and the instantaneous frequency (IF) were extracted as described above. The IA, IP, IF were normalized by the signal length in order to alleviate any biases due to different signals lengths and their histograms for 32 bins were calculated. As input vector to the three classifiers all 96 bins were used. Figure 2 shows the average of the three computed histograms for the normal Vs the pathogenic classes at $100 \%$ MVC. The similarity of the two curves demonstrates the difficulty of the problem. 


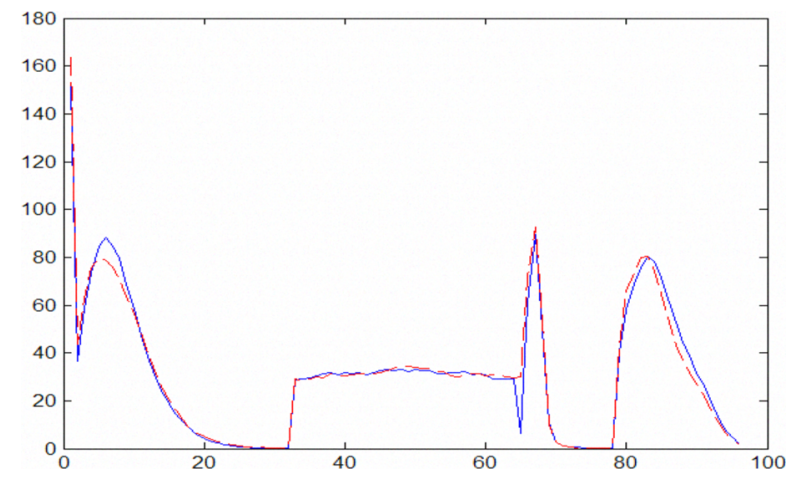

Fig. 2. The average of the three AM-FM computed histograms for the normal Vs the pathogenic (red dotted line) classes at $100 \%$ MVC.

Table I tabulates the correct classifications success rate for the three classifiers KNN, SOM and SVM and for the five force levels. In addition, the five force level scores per subject were combined with majority voting and the results are also given in Table I. For the KNN classifier the values provided in Table I are for $\mathrm{k}=11$ which gave the best results and for the SOM for a $7 \times 7$ map matrix and an evaluation neighborhood window $3 \times 3$ for the same reason.

Best classifier was by far the SVM with average success rate $72.5 \%$ compared to $59.5 \%$ for the $\mathrm{KNN}$ and $58.0 \%$ for the KNN classifier. Best force levels were the $10 \%$ MVC with average success rate $69.2 \%$ and the $100 \%$ MVC with $66.7 \%$ respectively. These results need further investigation and interpretation. Best individual result was $80 \%$ with the SVM classifier at $10 \%$ MVC.

Combining the five force level scores per subject with majority voting improved the average success rate, reaching in the case of the SVM classifier from $72.5 \%$ to $77.5 \%$. Combining all the outputs from all the classifiers gave $62.5 \%$. From the three AM-FM features, the best was the IF feature with average success rate $68.0 \%$ followed by the IA with $55.0 \%$ and the IP with $53.5 \%$.

TABLE I

CORRECT CLASSIFICATIONS RATE IN \% PER CLASSIFIER, PER FORCE LEVEL AND WHEN THE FIVE FORCE LEVEL SCORES WERE COMBINED USING MAJORITY VOTING.

\begin{tabular}{|ccccc|}
\hline \hline Force Level & KNN & SOM & SVM & Average \\
\hline $10 \%$ & 72.5 & 55.0 & 80.0 & 69.2 \\
\hline $30 \%$ & 50.0 & 60.0 & 75.0 & 61.7 \\
\hline $50 \%$ & 60.0 & 57.5 & 67.5 & 61.7 \\
\hline $70 \%$ & 52.5 & 55.0 & 65.0 & 57.5 \\
\hline $100 \%$ & 62.5 & 62.5 & 75.0 & 66.7 \\
\hline Average & 59.5 & 58.0 & 72.5 & 63.5 \\
\hline & & & & \\
\hline Combined & 57.5 & 60.0 & 77.5 & 65.0 \\
\hline \hline
\end{tabular}

\section{CONCLUDING REMARKS}

In previous work [5], [6] it was shown that time and frequency domain features extracted from the SEMG signals can be used successfully for the classification and the identification of neuromuscular disorders. In this work it was shown that AM-FM representations provide new feature sets, which can be used successfully for the classification of SEMG signals with a high success rate comparable to results obtained when using needle EMG data [3], [4]. This can be considered as the main contribution of this work showing that SEMG can be used as a non-invasive alternative to needle EMG for the detection of neuromuscular disorders.

Additionally in future work, cumulative density functions (CDF) and probability density functions (PDF) can be extracted from the AM-FM representations and used for classification instead of the histograms. It remains in future work to directly compare the traditional features with the new AM-FM features using the same dataset. Also more efficient neural classifiers like the SVM can provide the tool for designing a successful SEMG diagnostic system.

\section{REFERENCES}

[1] Merletti R, De Luca C.J. "New techniques in surface electromyography, in Computer aided electromyography and expert systems," edited by Desmedt J.E., Elsevier, vol. 2, Amsterdam-New York-Oxford, Chapter 9, section 3, pp. 115-124, 1989.

[2] Farina D, Fosci M, Merletti R, "Motor unit recruitment strategies investigated by surface EMG variables," Journal of Applied Physiology, 92, pp. 235-247, 2002.

[3] Abel E.W, Zacharia P.C., Forster A., Farrow T.L., "Neural network analysis of the EMG interference pattern," Medical Engineering and Physics, 1(1): pp. 12-17, 1995.

[4] Christodoulou C.I., Pattichis C.S., Fincham W.F., "A Modular Neural Network Decision Support System in EMG Diagnosis," Journal of Intelligent Systems, Special issue on Computational Intelligent Diagnostic Systems in Medicine, ed. by C.N. Schizas, Volume 8, Nos. 1-2, pp. 99-143, 1998.

[5] Abou-Chadi F.E.Z., Nashar A., Saad M., "Automatic analysis and classification of surface electromyography," Frontiers in Medical Biological Engineering, 1(1)1: pp. 13-19, 2001.

[6] Kaplanis P.A, "Surface Electromyography for the Assessment of Neuromuscular Disorders," Ph.D. Thesis, Kings College, University of London, 2004.

[7] Murray V., V. Rodriguez P., Pattichis M.S., "Robust Multiscale AMFM Demodulation of Digital Images," IEEE International Conference on Image Processing, vol.1, pp. 465-468, Oct. 2007.

[8] Pattichis M.S., Bovik A.C., "Analyzing image structure by multidimensional frequency modulation," IEEE Transactions on Pattern Analysis and Machine Intelligence, vol. 29, no. 5, pp. 753-766, 2000.

[9] Tou J.T., Gonzalez R.C., "Pattern Recognition Principles", AddisonWesley Publishing Company, Inc., 1974

[10] Kohonen T., "The Self-Organizing Map," Proceedings of the IEEE, Vol. 78, No. 9, pp. 1464-1480, Sept. 1990.

[11] Joachims T., "Making large-scale SVM learning practical," In: Schölkopf B, Burges C, Smola A (eds) Advances in kernel methodssupport vector learning, MIT, Cambridge, Chapter 11, 1999. 